\title{
Study on the Formation Control Methods for Multi-Agent Based on Geometric Characteristics
}

\author{
LI-LI He \\ Fair Friend Institute of Electromechanics \\ Hangzhou Vocational and Technical College \\ Hangzhou, 310018, China \\ e-mail: 153237697@qq.com
}

\author{
XIAO-CHUN LOU \\ Fair Friend Institute of Electromechanics \\ Hangzhou Vocational and Technical College \\ Hangzhou, 310018, China \\ e-mail: 1216389546@qq.com
}

\begin{abstract}
Multi-agent formation control is the process in which the teams formed by multiple agents move to specific target or specific direction. The formation method of the linear formation and circular formation are given in this paper, based on the geometric characteristics of the formation formed by multi-agent. The process in which 5 agents arrived at the designated target point and formed a linear formation is achieved through simulation; and 4 agents formed a circular formation and cooperated to carry heavy weights. The result of the three-dimensional simulation shows the feasibility of the method to form multi-agent formations in different environments and different tasks.
\end{abstract}

Keywords-Multi-agent; Cooperation; Formation, CLC: TP242 WM: A

\section{INTRODUCTION}

There are many researches and applications for agent at home and abroad. Various planning algorithms of obstacle avoidance movement are proposed. As the task demands that agents are required to accomplish become higher and higher, the difficulties of the production and control of a single agent would increase greatly if we still want to design and manufacture single agent to complete too complex tasks. If multi-agent is used to accomplish a complex task [1], the desired formation can be formed by multiple agents. Coordinating and cooperating with each other, each agent only needs to complete some action that is simple and easy to control. Thus not only the complex problems can be simplified, but also the stability of the agent can be strengthened.

Multi-agent formation control is the study on the process in which the teams formed by multiple agents move to specific target or direction. Specific geometric formation is kept between multiple agents. In order to complete specific task requirements, adaptive adjustments are made for particular environmental constraints through formation transformation strategies. One or more reference points often need to be created during the process of multi-agent formation control. Each agent calculates its appropriate location in the formation according to its own reference point so that the system can engender the required geometry overall. Literature [2] described the selection of various reference points.
The methods for multi-agent formation control use the following two steps generally: Firstly, the target location of the agent is determined according to the current environment. Afterwards, the control command is generated according to the control strategy. The agent is driven to form certain formations. At the same time, it moves to the target stably. To sum up, they can be divided into the following three categories [3]: The leader follower method [4], the behavior based method [5] and the virtual structure method [6]. Yun, etc. [7] carried out a deep study on the two-dimensional multi-robot formation of the linear and circular formations. "Swarm-bot" is a great example of multi-agent collaboration. Nouyan etc. [8] studied the self-assembly of this kind of agent and the problem of multi-agent queue formation. Soheil etc. [9] made analysis about the constraints for multi-robot formation control in regard to theory and application.

Detailed description is given in this paper to represent the formation methods of the linear formation and circular formation, based on the geometric characteristics of the formation formed by multi-agent.

\section{MathematicAl Description OF Multi-AgENT FORMATION}

Firstly, the locations of different agents need to be described in the process of multi-agent formation control. The agent is usually simplified for a particle when describing the mutual position of multi-agent. In addition, the positional relationship between these agents and the center points of the desired formations need to be described. The center point of the formation is assumed to be $O\left(x_{c}, y_{c}, \boldsymbol{\theta}_{c}\right)$. Where, $\left(x_{c}, y_{c},\right)$ is the position of the center point of the formation in the global coordinate system. $\theta_{c}$ is the forward direction of the formed formation. The positional relationship between other agents' positions $\left(x_{i}, y_{i}, \theta_{i}\right)$ and the center points of the formations can be expressed by polar coordinates $(l c i, \varphi c i)$. Since the forward direction of each agent needs to meet certain requirements in order to maintain the neatness of the formation in the motor process of the formation, the position of the $i^{\text {th }}$ robot can be calculated as:

$$
\left\{\begin{array}{l}
x_{i}=x_{c}+1_{c i} \cos \varphi_{c i} \\
y_{i}=y_{c}+1_{c i} \sin \varphi_{c i}
\end{array}\right.
$$




\section{Description OF Multi-AgEnt Formation}

The distance between the two agents not only need to meet the requirements of non-interference, but also need to meet the requirements that the agents can't be in each other's detection range of obstacle avoidance. The peripheral radius of the $\mathrm{i}^{\text {th }}$ agent is set to $r_{0}^{i}=r_{0}$ and the detection range of obstacle avoidance of the agent is $r_{a}^{i}=r_{a}=D_{\min }^{i}$. The formation process of the linear formation and circular formation of $\mathrm{n}$ agents is discussed, based on geometric characteristics of the graphics.

\section{A. Formation of the Linear Formation}

Step 1: The $\mathrm{i}^{\text {th }}$ agent calculates the farthest agent $R^{f}\left(X g^{f}\right.$ $\left.Y g^{f} \quad Z g^{f}\right)$ and the nearest agent $R^{c}\left(\begin{array}{lll}X g^{c} & Y g^{c} & Z g^{c}\end{array}\right)$ from it among multiple agents according to sensor information. The distance between the two agent is $D_{R} f_{R}^{c}$.

Step 2: Judge the distance between the farthest agent $R^{f}$ and the nearest agent $R^{c}\left(D_{R} f_{R^{c}}\right)$. If $D_{R f_{R^{c}}<2 r_{a}}$, agent $R^{f}$ and agent $R^{c}$ move $v_{\max } t$ away from each other respectively; If $D_{R} f_{R^{c}} \geqslant 2 r_{a}$, calculate the distance $d$ between the $\mathrm{i}^{\text {th }}$ agent and the straight line $l$ formed by $R^{f}$ and $\mathrm{R}^{\mathrm{c}}$. The position of the foot point $\mathrm{p}$ is determined. And judge whether the foot of the agent is located between $R^{f}$ and $R^{c}$ or not.

Step 3: If the foot of the $\mathrm{i}^{\text {th }}$ agent is located between $R^{f}$ and $R^{c}$ and $D_{R^{f} R^{c}} \geqslant 4 r_{0}$, the segment between $R^{f}$ and $R^{c}$ is divided into $n-1$ aliquots equally. The $\mathrm{i}^{\text {th }}$ agent moves to the division point $p_{m}$.

Step 4: If the foot of the $\mathrm{i}^{\text {th }}$ agent is not located between $R^{f}$ and $R^{c}$ or $2 r_{a} \leqslant D_{R^{f} R^{c}}<4 r_{0}$, the $\mathrm{i}^{\text {th }}$ agent moves to the point that is $d$ away from the nearest agent $R^{c}$ and located in the opposite direction of the farthest agent $R^{f}$ on straight line $l$. Followed by cycle, thus the $\mathrm{n}$ agents can form the linear formation.

\section{B. Formation of the circular formation}

1) The central position of multi-agent system is determined according to each agent's own position. Suppose there are $n$ same agents $R_{i}(i=0, \cdots \cdots, n)$. The position of the agent can be simplified as $R_{i}$ $\left(X_{i}, Y_{i}, \theta_{i}\right)$. Thus the position of the center of gravity for the entire multi-agent system $G\left(X_{g}, Y_{g}\right)$ can be given by:

$$
X_{g}=\sum_{1}^{n} X_{i} \quad Y_{g}=\sum_{1}^{n} Y_{i}
$$

2) The target location of each agent $T_{i}$ is determined according to the obtained position of the center of gravity and the desired formation. The steps are as follows:

a. The expression to calculate the circular formation: For the circular formation of radius $r$, its expression is set as $F 1$. So:

$$
F 1:(X-X g)^{2}+(Y-Y g)^{2}=r^{2}
$$

b. Evaluate the agent that is nearest to the formation $R_{m}\left(X_{m}, Y_{m}\right)$. Suppose the nearest agent is $R_{j}$, so:
$X_{m}=X_{j}, \quad Y_{m}=Y_{j}$

c. The target location of the nearest agent $T_{m}$ is obtained according to the coordinate of the nearest agent. The linear equation of the connecting line between the center of gravity of the agent and the coordinate of the nearest agent is set as $F_{j}$ :

$$
\frac{y-Y_{m}}{Y_{g}-Y_{m}}=\frac{X-X_{m}}{X_{g}-X_{m}}
$$

The target location of the nearest agent $T_{m}$ can be acquired according to formula (3) and (4).

d. Evaluate the target location of other agents $T_{i}$.

In counterclockwise rotation, the target location of the $\mathrm{k}^{\text {th }}$ agent after the nearest agent $R_{m}$ is set as $T_{m+k}$. The linear equation of the connecting line between the center of gravity and the agent is set as $F_{j+k}$. We have:

$$
y=Y_{g}+\tan \left(\arctan \left(\frac{Y_{g}-Y_{m}}{X_{g}-X_{m}}\right)+\frac{2 \pi}{n} k\right) \cdot\left(x-X_{g}\right)
$$

The target location of any agent after the nearest agent $R_{m}$ is obtained according to formula (3) and (5). The transformation of the circular formation is completed.

\section{IV.Simulation FOR MULTI-AGENT FORMATION CONTROL}

\section{A. Simulation for Multi-agent Queuing Behavior}

In order to verify the feasibility of the above analysis, the webots simulation software is adopted to simulate the behavior that the system formed by 5 agents coordinated to form the linear formation. Each agent began to move from different locations (Figure 1a); the agents coordinated to reach the target point (Figure $1 \mathrm{~b}$ ); the agents adjusted their respective gestures and connected into rectilinear queue (Figure 1c).

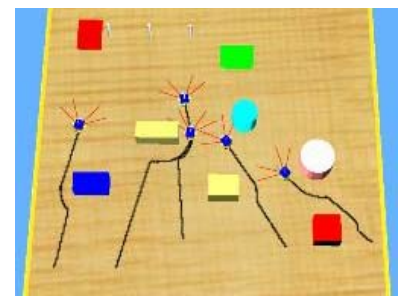

(a). The initial state of the simulation

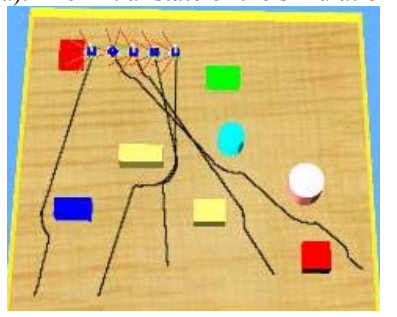

(b). Moved towards the target point 


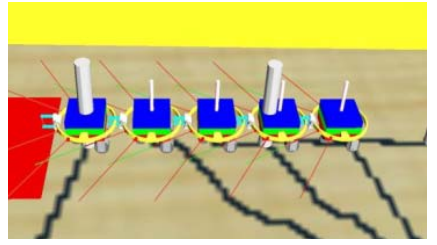

(c). The agents coordinated to complete the linear formation Figure 1. The formation process of multi-agent linear formation

\section{B. Simulation for the forming of the circular formation}

Four agents were distributed in the environment randomly. The target object (the handling object) was outside the detection range of all the agent sensors. The target location was the left direction of Figure 2, as the " $X$ " shown in Figure 2a. The target object would be found with the movement and exploration of the agents. The agent who first discovered the object moved towards the direction of the target object. At the same time, the sensor detected the object and completed its positioning, as shown in Figure $2 b$. The agent signaled through its own communication equipment to tell the other agents the position of the target object. Subsequently, three other agents coordinated to move towards the target object. Then the circular formation was formed, as shown in Figure 2c. Finally, the 4 agents who surrounded a circle handled the target object to the target position jointly.

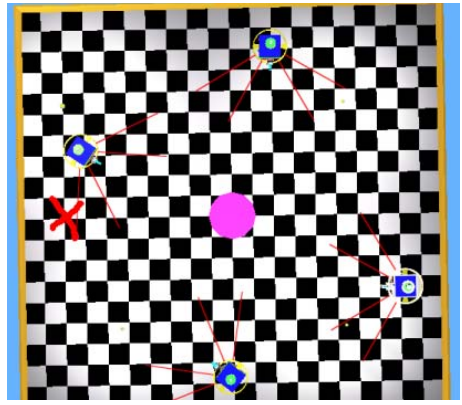

(a) The initial state of the simulation

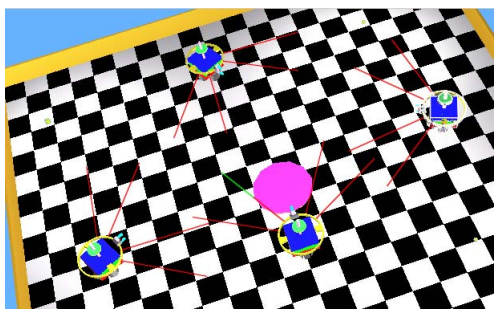

(b) Moved towards the target point

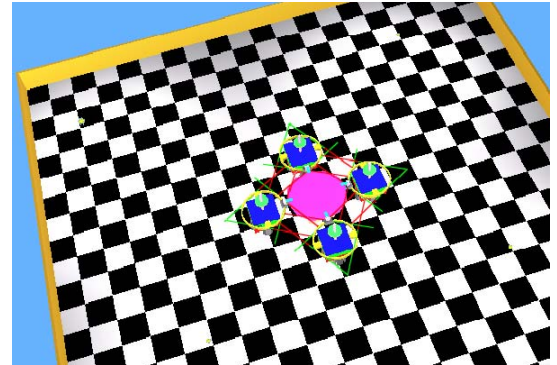

(c) The agents coordinated to complete the circular formation

Figure 2 Multiple agents formed the circular formation and coordinated to carry the heavy weights.

\section{V.CONCLUSION}

The formation control method for multi-agent formation is analyzed in this paper. The formation method of the linear formation and circular formation are given in this paper, based on the geometric characteristics of the desired multi-agent formations. Finally, the following process is achieved through simulation: 5 agents arrived at the designated target point and formed a linear formation as well as 4 agents collaborated to form a circular formation and carried heavy weights. The simulation results show the effectiveness of the method.

\section{ACKNOWLEDGMENTS}

This work was supported in part by Zhejiang province foundation of nature and science through project Y1101355.

\section{REFERENCES}

[1] Chen Jianping, Yang Yimin. Research of the systemic motion and coordination for multiple mobile robots. Computer engineering and applications, 2009, 45(23):44-49.

[2] Balch T, Arkin R C. Behavior-based formation control for multirobot teams. IEEE Trans. Robot. Automat. 1998, 14(6): 926-939.

[3] Su Zhibao, Lu Biaolian. The formation control method of multiple mobile robots[J]. Rorbot, 2003, 25(1):88-91

[4] Das A K. A vision-based formation control framework. IEEE Transaction on Robotics and Automation. 2002, 18(5): 813-825.

[5] Lawton J R T, Beard R W, Young B J. A Decentralized Approach to Formation Maneuvers. IEEE Transactions on Robotics and Automation.2003, 19(6): 933-941.

[6] Egerstedt M, Hu X. Formation Constrained Multi-Agent Control. IEEE Transactions on Robotics and Automation. 2001. 17(6): 947-951.

[7] Xiaoping Y, Gokhan A, Okay A. Line and circle formation of distributed physical mobile robots[J]. Journal of Robotic Systems, 1997, 14(2):63-76.

[8] Nouyan S, Groß R, Bonani M, Mondada F, et al. Group transport along a robot chain in a self-organized robot colony $[\mathrm{C}] / /$ Proceedings of the 9th International Conference on Intelligent Autonomous Systems. Amsterdam, The Netherlands: IOS Press, 2006 , 433-442.

[9] Soheil Keshmiri and Shahram Payandeh. A Centralized Framework to Multi-Robots Formation Control: Theory and Application[J]. Lecture Notes in Computer Science (LNCS), Springer, 2011,Vol. 6066, $85-98$ 